\title{
55. SEDO ANGLICI-THYMETUM CAESPITITII, NUEVO PASTIZAL PIONERO VIVAZ DE LOS SUELOS ESQUELÉTICOS DEL SUROESTE DE GALICIA
}

\author{
Juan RODRÍGUEZ OUBIÑA, Santiago ORTIZ e Íñigo PULGAR
}

Sedo anglici-Thymetum caespititii, a new perennial pasture of the mobile rock slides of the SW of Galicia

Palabras clave. Thymus caespititius, Koelerio-Corynephoretea, pastizales, fitosociología, Galicia, España.

Key words. Thymus caespititius, Koelerio-Corynephoretea, pastures, phytosociology, Galicia, Spain.

En el occidente de Galicia los pastizales pioneros vivaces que se desarrollan sobre suelos esqueléticos o en arenales costeros se encuentran mucho más diversificados que los de zonas más septentrionales de Europa occidental (cf. Braun-Blanquet \& Tüxen 1952; Géhu J.- M.; Géhu, J. \& Caron 1978; Clement \& Touffet 1978; etc.), suelen ser bastante más ricos en endemismos y colonizar áreas mucho más amplias. De este tipo de vegetación tan interesante se posee escasa información de Galicia, y, en general, de las áreas colinas del noroeste peninsular, a las que recientemente hicieron referencia Díaz González y Fernández Prieto (1994), por lo que decidimos estudiarla con detalle en el territorio gallego. En el presente trabajo se dan a conocer los primeros datos sobre los pastizales presididos por Thymus caespititius.

Para el estudio de estos tomillares se ha empleado el método fitosociológico de la escuela de Zürich-Montpellier (Braun-Blanquet 1964) y para la descripción de los sintaxones se ha seguido el Código de Nomenclatura Fitosociológica (Barkman et al. 1986). La caracterización biogeográfica y bioclimática se ha realizado siguiendo la propuesta de RivasMartínez (1987).
La nomenclatura de las fanerógamas citadas en el tex to y en la tabla 1 es coincidente con la de Flora Europaea (Tutin et al. 1964 1980) o, en el caso de los taxones incluídos en los volúmenes publicados hasta la fecha, con la de Flora Iberica (Castroviejo et al. 19681993), con la excepción de las siguientes: Agrostis truncatula Parl. subsp. commista Castroviejo \& Charpin, Centaurea corcubionensis Laínz, Conopodium majus (Gouan) Loret subsp. ramosum (Costa) Silvestre y Simethis mattiazzi (Vandelli) Saccardo. La nomenclatura de los musgos está en concordancia con la empleada por Smith (1980), con la excepción de Racomitrium elongatum Frisvoll.

Sedo anglici-Thymetum caespititii RodríguezOubiña, Ortiz et Pulgar ass. nova

Holotypus: inventario $n^{0} 2$, tab. 1

Asociación caracterizada básicamente por la coexistencia de Thymus caespititius, Agrostis truncatula subsp. commista, Sedum brevifolium, Sedum anglicum y Conopodium majus subsp. ramosum. Entre las compañeras predominan plantas anuales (que compiten por el mismo medio ecológico), musgos (cuyo óptimo se encuentra en suelos todavía menos profundos) y componentes de los brezales

El presente trabajo ha sido financiado por la Consellería de Educación e Ordenación Universitaria de la Xunta de Galicia, a través del proyecto XUGA $20315 \mathrm{~B} 94$. 


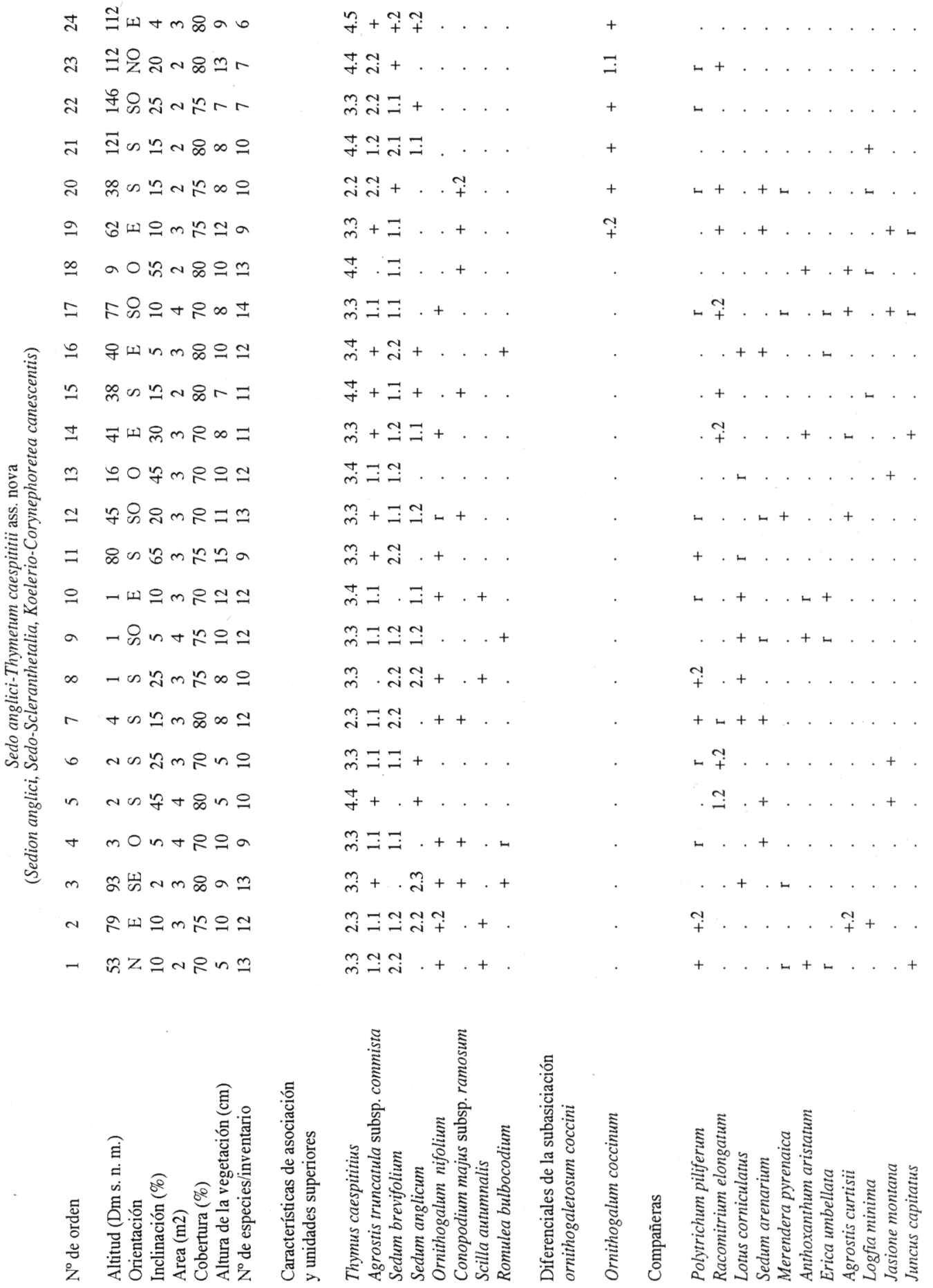




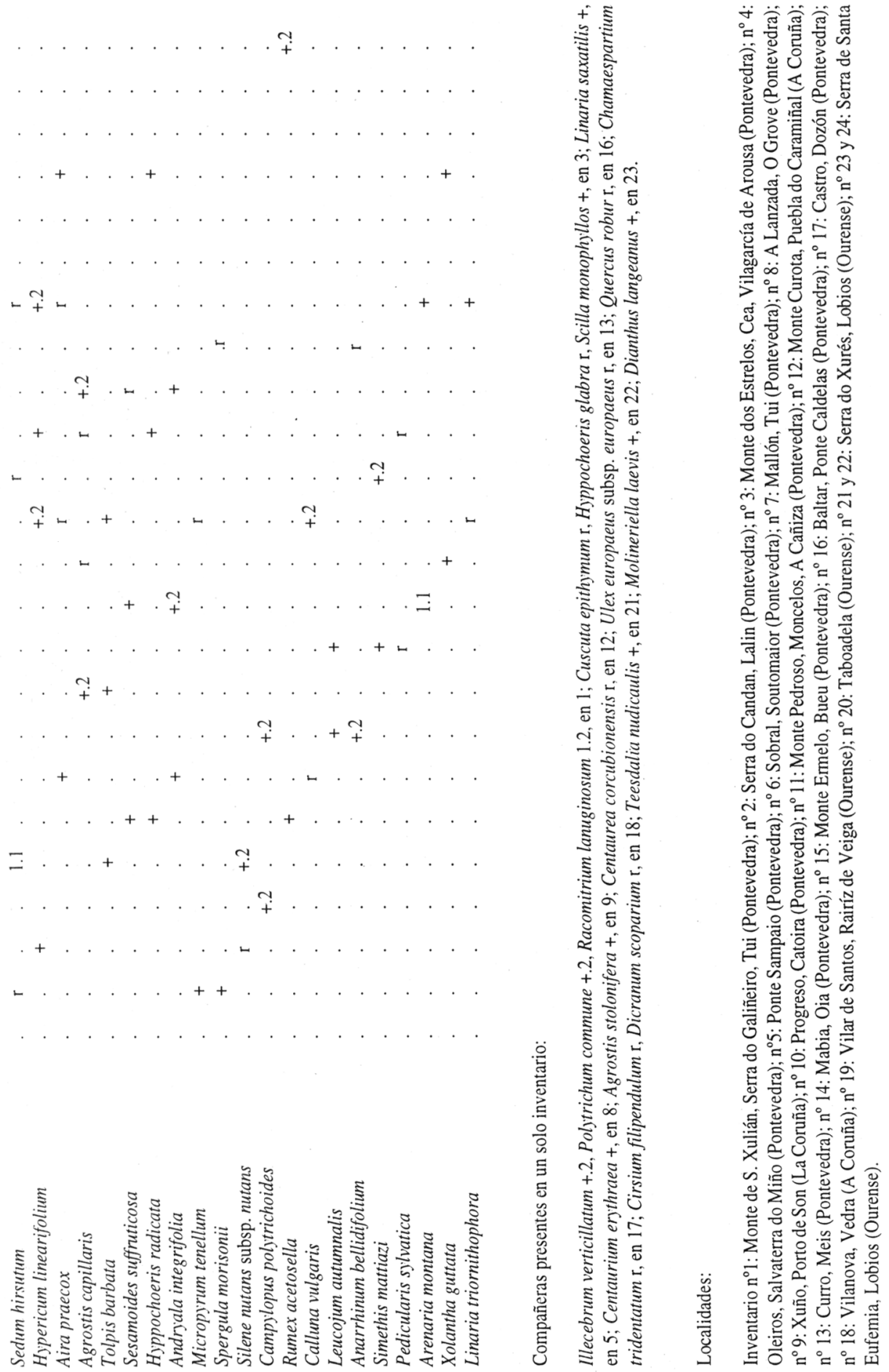


adyacentes (ver ecología).

Teniendo en cuenta la composición florística de esta asociación y su emplazamiento bioclimático (básicamente a nivel del piso colino), debe incluirse en la alianza y orden atlánticos Sedion anglici Br.- Bl. in Br.- Bl. \& Tüxen 1952 y Sedo-Scleranthetalia Br.- B1. 1955 respectivamente, de la clase KoelerioCorynephoretea Klika in Klika \& Novak 1941. Obviamente las diferencias florísticas de la Sedo anglici-Thymetum caespititii respecto a sus homólogas de Sedion anglici de zonas más septentrionales del continente europeo son destacables; dicha asociación es mucho más rica en caméfitos y hemicriptófitos representativos de este tipo de vegetación, la mayoría endémicos peninsulares, entre los que destacan: Thymus caespititius, Agrostis truncatula subsp. commista, Ornithogalum unifolium y Conopodium majus subsp. ramosum. Estos taxones estan ausentes incluso en las comunidades de Sedion anglici más afines como la asociación irlandesa Airo praecocis-Sedetum anglici $\mathrm{Br}$-Bl. \& Tüxen 1952 o las bretonas Dactylo marinae-Sedetum anglici (G. Lemée 1938) J.-M. Géhu, J. Géhu \& B. C. Caron 1978, Festuco trachyphyllaeSedetum anglici Clement \& Touffet 1978 y Festuco tenuifoliae-Sedetum anglici Clement \& Touffet 1978, en las que la única especie característica coincidente es Sedum anglicum. Las afinidades de la Sedo-Thymetum con las comunidades de la alianza Sedion pyrenaici Tüxen ex Rivas-Martínez, T. E. Díaz, F. Prieto, Loidi y Penas in T. E. Díaz y F. Prieto 1994 de las altas montañas septentrionales y occidentales de la Península Ibérica también son escasas. La asociación más próxima es Agrostio durieui-Sedetum pyrenaici RivasMartínez, T. E. Díaz, F. Prieto, Loidi y Penas 1984, mucho más pobre en especies que la Sedo-Thymetum (sus taxones más representativos son Sedum anglicum, $S$. brevifolium y Agrostis truncatula subsp. commista), y en ella estan ausentes Thymus caespititius, Ornithogalum unifolium, Conopodium majus subsp. ramosum, etc. y son frecuentes otras especies propias de su emplazamiento biogeográfico (Paronychia polygonifolia, Festuca eskia, etc.) que estan ausentes en la asociación que describimos. Asimismo, la relación de esta asociación con la alianza Hieracio castellani-Plantaginion radicatae Rivas-Martínez y Cantó 1987 (de óptimo supramediterráneo) es muy escasa ya que estan ausentes la práctica totalidad de las especies que caracterizan a esta última (cf. Rivas-Martínez y Cantó 1987).

En lo que respecta a la variabilidad de la Sedo anglici-Thymetum caespititii, hasta el momento se reconocen dos subasociaciones que tienen destinto areal. La subasociación thymetosum caespititii nova (inventarios 1-18, tab. 1), en la que habitualmente está presente Ornithogalum unifolium (holotypus: el mismo que el de la asociación, inventario ${ }^{0} 2$, tab. 1), se encuentra en las áreas más oceánicas del Suroeste de Galicia (provincia de Pontevedra, extremo sur de la provincia de La Coruña y extremo noroccidental de la provincia de Ourense). En la subasociación ornithogaletosum coccini nova (inventarios 19 24) Ornithogalum unifolium es sustituído por su vicariante Ornithogalum coccinum (holotypus inventario $n^{\circ} 23$, tab. 1). Esta última está ubicada en zonas más continentales del sur de la provincia de Ourense.

En lo referente a la fisonomía, esta asociación posee un aspecto muy característico y fácilmente reconocible durante todo el año debido a la presencia de Thymus caespititius que es un tomillo decumbente que con frecuencia se encuentra formando densas colonias. Esto es debido a su particular sistema de multiplicación vegetativa mediante la contínua emisión de raíces adventicias desde sus tallos rastreros. La comunidad es particularmente llamativa al principio del verano, período durante el cual tiene lugar la masiva floración de este tomillo y sus 
tonalidades rojizas contrastan con las blancas de otros constituyentes tales como Sedum brevifolium, S. anglicum y Ornithogalum unifolium. La cobertura de estos pastizales suele ser considerablemente elevada (por encima del $70 \%$ ) y su altura media es de unos $10 \mathrm{~cm}$.

La Sedo anglici-Thymetum caespititii se sitúa generalmente en posiciones de cumbre o ladera alta, colonizando litosuelos de perfil $\mathrm{AR}$, cuyo horizonte orgánico-mineral no suele sobrepasar los $10 \mathrm{~cm}$ de espesor y contacta directamente con la roca sin alterar o poco alterada. Incluso se observa en ocasiones esta comunidad entre las grietas de las rocas o en piedras recubiertas con un espesor de tierra insignificante $(2-3 \mathrm{~cm})$. Suele ser por tanto la última asociación cormofítica de la toposecuencia de vegetación, de forma que hacia zonas menos profundas cede paso a comunidades briofíticas de Polytrichetalia piliferi Hübschmann 1967 y a veces el tránsito es directo a comunidades epilíticas del Hedwigion ciliatae Phillipi 1956. Por el contrario hacia suelos más profundos, la Sedo anglici-Thymetum caespititii es sustituída por las facies más xéricas del brezal (Ulici europaei-Ericetum cinereae Bellot 1949, Ulici minoris-Ericetum umbellatae $\mathrm{Br}$.- Bl., P. Silva y Rozeira 1964 em. Rivas-Martínez 1979, fundamentalmente). En las proximidades a taludes y zonas de umbría con cierta humedad edáfica, en ocasiones se observan ecotonías con comunidades de Asplenietea trichomanis (Br.- Bl. in Meier \& Br.- Bl. 1934) Oberdorfer 1977, lo que explica la aparición de especies como Sedum hirsutum, Sesamoides suffruticosa, Anarrhinum bellidifolium, etc.

Debe destacarse asimismo que en el lugar donde se establece esta asociación presentan un desarrollo óptimo las comunidades de Helianthemetea gutati (Br.- Bl. in Br.- Bl. et al. 1952) Rivas Goday y Rivas-Martínez 1963 (Airo-Sedetum arenarii Izco, Guitian y Amigo 1986 , etc.) por lo que especialmente durante la primavera cohabitan plantas anuales y perennes

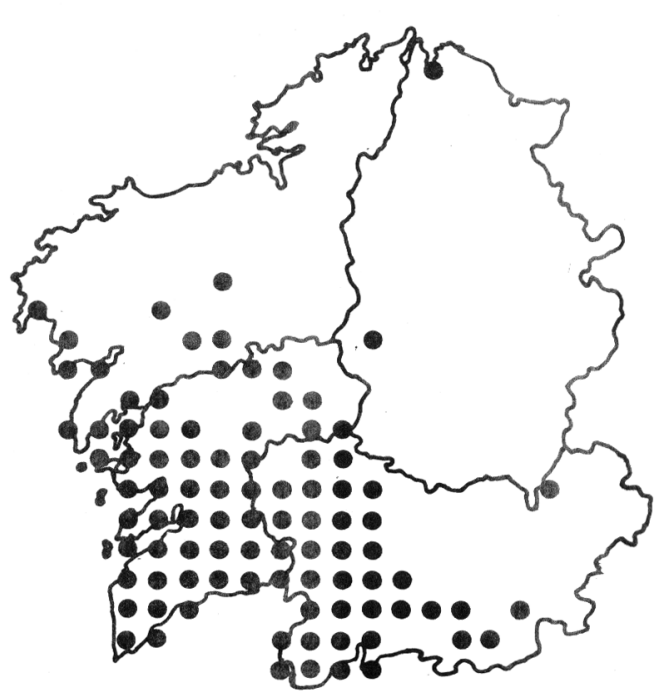

Figura 1. Distribución de Thymus caespititius en Galicia. Distribution of Thymus caespititius in Galicia

pertenecientes a ambas clases, lo que justifica la presencia de las primeras en la tabla 1.

En Galicia la asociación se ha observado indistintamente sobre sustratos ácidos y básicos (granitos diversos, esquistos pelíticos metamórficos, grauvacas, diques capa-básicos, gneises hiperalcalinos, anfibolitas, etc.). En las rocas ultrabásicas es sustituída por comunidades del Hieracio castellanaePlantaginion radicatae Rivas-Martínez y Cantó 1987 (Sagino merinoi-Plantaginetum radicatae Rodríguez-Oubiña y Ortiz 1991 subass. asteretosum aragonensis Rodríguez-Oubiña y Ortiz 1991, principalmente). La subasociación ornithogaletosum coccini se encuentra en medios análogos a los de la subasociación típica (thymetosum caespititii).

En lo que respecta a la distribución y delimitación biogeográfica de esta asociación, debe tenerse en cuenta que Thymus caespititius 
es una especie endémica de distribución considerablemente amplia (oeste de la Península Ibérica, Madeira y Azores), que se encuentra en zonas de diferente adscripción biogeográfica y bioclimática. En la Península Ibérica su distribución se localiza básicamente en la franja costera comprendida entre la provincia de Pontevedra y Beira Alta y Litoral, adentrándose tan solo unos $150 \mathrm{Km}$ hacia el interior en el límite entre Salamanca y Cáceres (cf. Morales 1986).

En Galicia Thymus caespititius está presente en el cuadrante suroccidental, en altitudes comprendidas entre el nivel del mar y los $1.500 \mathrm{~m}$., y penetra considerablemente hacia el interior en el extremo sur de la provincia de Ourense (en la figura 1 se indica la distribución conocida de este taxón en virtud de citas bibliográficas y observaciones propias). La distribución de la Sedo-Thymetum caespititii se corresponde en buena medida con el área reseñada anteriormente, extendiéndose al tercio noroccidental portugués y con la excepción del interior de la provincia de Ourense donde, posiblemente debido al clima de caracter mediterráneo más marcado, el comportamiento ecológico de Thymus caespititius es diferente ya que tiende a colonizar claros del brezal y muy raramente se encuentra en los suelos esqueléticos coexistiendo con los otros taxones que integran la comunidad.

En virtud de los datos anteriormente expuestos, en el territorio gallego esta asociación se encuentra emplazada fundamentalmente en el piso colino (horizontes termocolino y eucolino) del subsector Miñense, extendiéndose hacia el extremo suroccidental del Lucense (horizontes eucolino y colino superior) y el área occidental del subsector Juresiano-Queixense (horizonte colino superior, alcanzando ocasionalmente el piso montano), subsectores pertenecientes al sector Galaico-Portugués, provincia CántabroAtlántica y región Eurosiberiana. En los subsectores Miñense y Lucense predomina la subass. típica (thymetosum caespititii), propia de áreas oceánicas, y en el Juresiano-Queixense la subass. ornithogaletosum coccini.

El dinamismo de la Sedo angliciThymetum caespititii suele ser muy lento, pues, aunque continuamente es colonizada esta comunidad por especies de mayor biomasa (Ulex europaeus, Erica umbellata, etc.), éstas no soportan la sequía estival en suelos tan poco profundos y terminan muriendo. La penetración del brezal adyacente ocurre al incrementarse el espesor del suelo, sobre todo en litosuelos de cumbre o de ladera alta, y ésto tiene lugar con gran lentitud.

Por otro lado al estar integrada la comunidad por especies perennes, la dinámica de colonización de las áreas despejadas (claros del brezal, espacios quemados, etc.) es mucho menor que en el caso de los pastizales pioneros terofíticos (Airo praecocis-Sedetum arenarii) e incluso que los brezos y tojos, de manera que estos últimos terminan impidiendo que la SedoThymetum caespititii llegue a desarrollarse en estos medios y queda relegada a los suelos esqueléticos.

La frecuencia de incendios, especialmente en la provincia de Pontevedra, está provocando una importante regresión de las poblaciones de Thymus caespititius en zonas donde esta especie era muy abundante. Esto es debido a que esta planta de naturaleza cespitosa y con esencias volátiles arde con mucha facilidad y posteriormente no suele recuperarse. Los restantes componentes de la asociación son generalmente mucho menos afectados y su recuperación tiene lugar con rapidez. Debido a ésto es frecuente en zonas afectadas por incendios la aparición de representantes fragmentarios de asociación en los que no está presente el tomillo.

\section{BIBLIOGRAFÍA}

BRAUN-BLANQUET, J. -1964- Pflanzensoziologie. Grundzüge der Vegetationskunde. 3. Aufl. Wien. BRAUN-BLANQUET, J. \& R. TÜXEN -1952Irische Pflanzengesellschaften. Veroff. Geobot. Inst. Rübel, Zurich, 25: 224-415. 
CASTROVIEJO, S., M. LAÍNZ, G. LÓPEZ GONZÁLEZ, P. MONTSERRAT, F. MUÑOZ GARMENDIA, J. PAIVA y L. VILLAR -1986/ 1993- [eds.] Flora Iberica. C.S.I.C. Madrid.

CLEMENT, B. \& J. TOUFFET -1978- Les pelouses xerophiles autour de quelques affleurements schisteux en Bretagne interieure. Colloques Phytosociologiques, 6: 177-189.

DÍAZ GONZÁLEZ, T. E. y J.A. FERNÁNDEZ PRIETO -1994- La vegetación de Asturias. Itinera Geobot., 8: 243-528.

GÉHU, J.M., J. GÉHU \& B. CARON -1978- Les groupements á Sedum anglicum des falaises Nord-Armoricaines. Colloques Phytosociologiques, 6: 255-261.

MORALES, R. -1986- Taxonomía de los géneros Thymus (excluída la sección Serpyllium) y Thymbra en la península Ibérica. Ruizia, 3: (1324).

RIVAS-MARTÍNEZ, S. -1987-Memoria del mapa de las series de vegetación de España
(1:400.000). ICONA, Serie Técnica. Madrid.

RIVAS-MARTÍNEZ, S. y P. CANTÓ -1987- Datos sobre la vegetación de las sierras de Guadarrama y Malagón. Lazaroa, 7: 235-257.

SMITH, A. -1980- The moss flora of Britain and Ireland. Cambridge University Press. Cambridge.

TUTIN, T.G., V.H. HEYWOOD, N.A. BURGES, D.H. VALENTINE, S.M. WALTERS \& D.A. WEBB -(1964-1980)- [eds.] Flora europea. Vol. 1-5. Cambridge.

Aceptado para su publicación en Julio de 1996

Dirección de los autores. Dpto. Biología Vegetal. Laboratorio de Botánica. Facultad de Farmacia. Campus Universitario Sur. 15706, Santiago de Compostela

\title{
56. STIPION PARVIFLORAE ALL. NOVA, PASTIZALES VIVACES SUBNITRÓFILOS Y CALCÍCOLAS MEDITERRÁNEO-IBEROLEVANTINOS
}

\author{
Antonio DE LA TORRE, Francisco ALCARAZ y María VICEDO
}

Stipion parviflorae all. nova, perennial subnitrophilous and calcicolous mediterraneaniberolevantines grassland

Palabras clave: Fitosociología, ibero-levantino, Stipion parviflorae all. nova, Lygeo-Stipetea, pastizales.

Key words: Phytosociology, ibero-levantine, Stipion parviflorae all. nova, Lygeo-Stipetea, grassland.

Dentro de la clase Lygeo-Stipetea RivasMartínez 1978 se han venido reconociendo, desde que Rivas-Martínez (1978) propusiera el orden subnitrófilo Hyparrhenietalia hirtae, una serie de comunidades dominadas por hemicriptófitos herbáceos en las que abundan las gramíneas del género Stipa.

Estos pastizales viven sobre suelos poco profundos o muy alterados por acción antrópica o erosión, siempre en áreas térmicas, en los 